\title{
Dilated Anus
}

National Cancer Institute

\section{Source}

National Cancer Institute. Dilated Anus. NCI Thesaurus. Code C92767.

A characteristic sign of impending birth where the anal opening becomes wider due to increased pressure in the perineal region during the second stage of labor. 